\title{
Transversely Inhomogeneous Nonlinear Surface Ultrasonic Monopulses in Solid Film-Substrate System
}

\author{
M. Tecpoyotl-Torres*, G. Vera-Dimas, V. Grimalsky and S. Koshevaya \\ CIICAp, Autonomous University of State Morelos (UAEM), Cuernavaca Z.P. 62209, Mor., Mexico
}

(Received March 7, 2013; in final form December 17, 2013)

\begin{abstract}
Excitation of nonlinear acoustic monopulses, or baseband waves, is theoretically analyzed. The monopulses have a wide spectrum up till the gigahertz range $\leq 1 \mathrm{GHz}$. The propagation of waves is along the interface between a solid film and a solid substrate. A quadratic elastic nonlinearity in both contacting media of the system is taken into account. The nonlinear dynamics of waves depends essentially on the thickness of the film. The transverse inhomogeneity of the wave profile in the interface plane and the wave diffraction are taken into account, too. The baseband waves are not true solitons and manifest as single shock-like pulses in the case of an excitation from initial long rectangular pulses. The transverse inhomogeneity affects the dynamics of the shocks, and there is a possibility to focus the nonlinear monopulses.
\end{abstract}

DOI: $10.12693 /$ APhysPolA.125.1118

PACS: 43.25.Fe, 42.65.Tg, 62.50.Ef

\section{Introduction}

Last years, research of nonlinear wave phenomena in bounded solids has been of great interest [1-12]. Nonlinear wave effects in structures, which include thin solid films, are perspective for experimental observations due to a possibility to control linear and nonlinear wave properties. In this article the nonlinear surface acoustic waves in bounded solids have been investigated. This nonlinear wave phenomenon has applications in material research, microelectronics, sonochemistry, and signal processing.

The surface acoustic waves (SAW) possess attracting properties for observing nonlinear effects in the gigahertz range. The integral power levels, which are necessary for a manifestation of nonlinearity, are low. The combination of materials of the film and the substrate makes possible to control both the wave dispersion and the nonlinearity. It is possible to measure directly the magnitudes of the components of the velocity of SAW by acoustooptic methods.

Nonlinear SAW propagation in the layered solid systems was considered in many papers [3-12]. The most results of simulations were obtained within an approximation of moderate nonlinearity. In this case the wave was presented as a superposition of partial harmonics with slowly varying amplitudes. The transverse profiles of partial harmonics changed weakly, compared with the linear case. The baseband pulses, or monopulses, can be excited experimentally [8-10], but they are not true solitons, because they do not preserve their shapes after collisions $[6,10]$. The properties of nonlinear surface waves depend essentially on the combination of materials, the thicknesses of the films, and the methods of initial excitation.

*corresponding author; e-mail: tecpoyotl@uaem.mx
Below there is investigated the propagation of moderately nonlinear monopulses of SAW in the system solid thin film-substrate. The monopulses possess very wide spectrum that reach the gigahertz range $\leq 1 \mathrm{GHz}$. The transverse inhomogeneity in the plane of the film is taken into account, which leads to the wave diffraction. When the exciting force is monopulse-like, then the baseband nonlinear wave occurs, so the evolution of almost rectangular initial pulses is considered. It has been shown that true baseband solitons are absent in such a system.

The nonlinear waves are rather the single shocks. Under certain levels of initial intensities the nonlinearity ceases to be moderate. From the initial pulse of arbitrary duration only a single shock can be created. The transverse inhomogeneity influences essentially on the dynamics of nonlinear monopulses. It is possible to focus the nonlinear pulses and to observe the shocks from the initial pulses of small amplitudes. The properties of nonlinear waves depend on the thicknesses of the films. Namely, when the thickness of the film changes several times, from $0.1-0.2 \mu \mathrm{m}$ to $0.5-1 \mu \mathrm{m}$, the dynamics of the nonlinear pulse propagation can be changed qualitatively. In the case of thicker films the wave dispersion dominates, whereas in the thinner ones the nonlinearity can dominate.

\section{Basic equations}

The surface acoustic wave propagation in the system a thin solid film-solid substrate is under investigation. Isotropic contacting media are considered without slipping, i.e. the tight contact occurs between the media. Usually, the Lagrangian coordinate frame is used within the solid media [1, 3, 11-13]. The dynamic equation, i.e. the nonlinear elasticity theory one, for the mechanical displacement vector $\boldsymbol{u}$ in solids has the next form $[1,12,13]$ :

$$
\rho_{0} \frac{\partial^{2} u_{i}}{\partial t^{2}}=\frac{\partial \sigma_{i j}}{\partial x_{j}}, \quad \sigma_{i j}=\sigma_{i j}^{\mathrm{L}}+\sigma_{i j}^{\mathrm{NL}},
$$


where $\rho_{0}$ is the unperturbed density of a solid and $\sigma_{i j}$ is the Piola-Kirchhoff tensor. For an isotropic solid the expression of $\sigma_{i j}$ is $[1,2]$ :

$$
\begin{aligned}
\sigma_{i j} & =\rho_{0}\left(s_{l}^{2}-2 s_{\mathrm{t}}^{2}\right) \operatorname{div} \boldsymbol{u} \delta_{i j}+2 \rho_{0} s_{\mathrm{t}}^{2} u_{i j} \\
& +\left(\rho_{0} s_{\mathrm{t}}^{2}+A / 4\right)\left(\frac{\partial u_{i}}{\partial x_{l}} \frac{\partial u_{j}}{\partial x_{l}}+\frac{\partial u_{i}}{\partial x_{l}} \frac{\partial u_{l}}{\partial x_{j}}+\frac{\partial u_{l}}{\partial x_{i}} \frac{\partial u_{l}}{\partial x_{j}}\right) \\
& +B \frac{\partial u_{j}}{\partial x_{i}} \operatorname{div} \boldsymbol{u}+\left[\rho_{0}\left(s_{l}^{2}-2 s_{\mathrm{t}}^{2}\right)+B\right] \frac{\partial u_{i}}{\partial x_{j}} \operatorname{div} \boldsymbol{u} \\
& +(A / 4) \frac{\partial u_{j}}{\partial x_{l}} \frac{\partial u_{l}}{\partial x_{i}}+\left\{C(\operatorname{div} \boldsymbol{u})^{2}+\left[\rho_{0}\left(s_{l}^{2}-2 s_{\mathrm{t}}^{2}\right) / 2\right.\right. \\
& \left.+B / 2]\left(\frac{\partial u_{l}}{\partial x_{m}}\right)^{2}+(B / 2) \frac{\partial u_{l}}{\partial x_{m}} \frac{\partial u_{m}}{\partial x_{l}}\right\} \delta_{i j} .
\end{aligned}
$$

The quadratic nonlinear terms are taken into account here. The coefficients in nonlinear terms are combinations of both material nonlinear modules $(A, B, C)$ and the linear ones due to the geometric nonlinearity $[1,2]$. Here $s_{1, t}$ are longitudinal and transverse acoustic velocities; $u_{i}$ are the Cartesian components of the mechanic displacement. The results of the simulation presented below are of qualitative character and do not depend essentially on the values of nonlinear modules. It is assumed only that the magnitudes of nonlinear modules are comparable in the film and in the substrate. The wave dissipation due to viscosity, proportional to the square of frequency, is also taken into account, which is not presented in Eq. (1).

The ordinary boundary conditions at the interface film-substrate are used [2]; namely, the components of displacement vector $u_{i}$ and the normal components of the elastic force $\sigma_{i j} n_{j}$ are the same, where $\boldsymbol{n}=(1,0,0)$ is the normal vector to the unperturbed interface. The geometry of the system under consideration is described below. The axis $O Z$ is directed horizontally along the wave propagation in the interface plane, $O X$ one is directed vertically upwards, see Fig. 1a. The thickness of the film $(-h<x<0)$ is $h=$ const. The solid substrate occupies the space $x<-h$. The surface $x=0$ is free. In this system the linear SAW can propagate along $O Z$ axis with displacement components $\boldsymbol{u}=\left(u_{x}, 0, u_{z}\right)$. The linear dispersion equation for the sound waves has been obtained from subjecting the partial solutions of the linearized dynamic Eq. (1) in each region to the elastic boundary conditions.

The dispersion curve for the lowest acoustic surface mode is presented in Fig. 1b. The contact of the $\mathrm{Si}(\mathrm{Ge}) \mathrm{O}_{2}$ film with $\mathrm{Si}$ substrate is considered. The following parameters are used: $h=0.25 \mu \mathrm{m}, c_{\mathrm{t}}=$ $2.5 \times 10^{5} \mathrm{~cm} / \mathrm{s}, c_{1}=4.0 \times 10^{5} \mathrm{~cm} / \mathrm{s}, \rho_{1}=3.0 \mathrm{~g} / \mathrm{cm}^{3}$ $\left(\mathrm{Si}(\mathrm{Ge}) \mathrm{O}_{2}\right) ; s_{\mathrm{t}}=5.0 \times 10^{5} \mathrm{~cm} / \mathrm{s}, s_{1}=8.4 \times 10^{5} \mathrm{~cm} / \mathrm{s}$, $\rho_{2}=2.33 \mathrm{~g} / \mathrm{cm}^{3}(\mathrm{Si})$, where $c_{\mathrm{l}, \mathrm{t}}, s_{\mathrm{l}, \mathrm{t}}$ are longitudinal and transverse bulk velocities in the film and the substrate, respectively; $\rho_{1,2}$ are the mass densities. A crystalline anisotropy in $\mathrm{Si}$ is neglected here. These waves possess an essential dispersion [2]. One can see that the wave dispersion in the frequency ranges $\omega<\omega_{\text {crit }}$ and

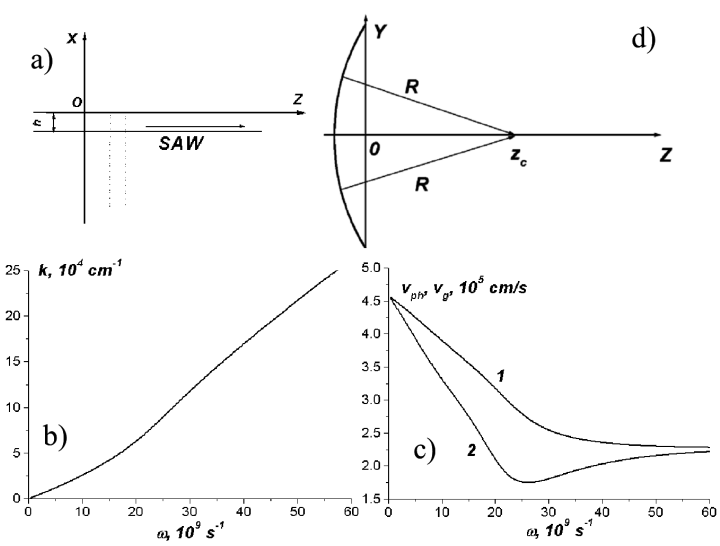

Fig. 1. Part (a) is the geometry of the problem. The film is at $-h<x<0$, the substrate is at $x<-h$. The free surface is at $x=0$. Part (b) is the linear wave dispersion for SAW in the layered solid system filmsubstrate where $\omega_{\text {crit }}=2.62 \times 10^{10} \mathrm{~s}^{-1}$ for used parameters of $\mathrm{Si}(\mathrm{Ge}) \mathrm{O}_{2}$ film-Si substrate. Part (c) is the corresponding dependences of the phase velocity (curve 1 ) and the group (curve 2) one on the frequency. The thickness of the film is $h=0.25 \mu \mathrm{m}$. Part (d) is the focusing of SAW in the surface plane $Z O Y$ by means of the curvilinear antenna.

$\omega>\omega_{\text {crit }}$ differs from each other. The value of $\omega_{\text {crit }}$ is $\omega_{\text {crit }}=2.62 \times 10^{10} \mathrm{~s}^{-1}$ for the used parameters of $\mathrm{Si}(\mathrm{Ge}) \mathrm{O}_{2}$ film-Si substrate. Because $\omega_{\text {crit }}$ depends on the thickness of the film, the pulse dynamics is different for the structures with different thicknesses of the film. Despite the presence of the wave dispersion and nonlinearity, the SAW monopulses do not behave as true solitons, as demonstrated below.

\section{Baseband nonlinear waves}

The elastic nonlinearity is assumed as moderate. This gives a possibility to use the spectral method for a consideration of the baseband wave dynamics. It means that the localized nonlinear wave can be presented as the set of harmonics with slowly varying amplitudes $A_{j}(z, y)$, but the transverse profiles $\boldsymbol{f}_{j}(x)$ of the harmonics are almost the same as in the linear case [6-8]:

$$
\boldsymbol{v} \equiv \frac{\partial \boldsymbol{u}}{\partial t}=\frac{c_{\mathrm{t}}}{2} \sum_{j} A_{j}(z, y) \boldsymbol{f}_{j}(x) \mathrm{e}^{\mathrm{i} \omega_{j} \eta}+\text { c.c. }+\delta \boldsymbol{v},
$$

where $\omega_{j}=(2 \pi / T) j(j=1, \ldots, N), \eta=t-z / v_{0}$ is the "running time" in the moving coordinate frame, $v_{0}=\left.(\partial \omega / \partial k)\right|_{k=0}$. The value $T$ is the temporal domain where the dynamics of localized nonlinear pulse occurs. With respect to the variable $\eta$ the periodical boundary conditions are applied. The excitation takes place at $z=0$. The additional term $\delta \boldsymbol{v}$ can be obtained analogously to the theory of the excitation of electromagnetic waveguides, namely, by means of imaginative elimination of nonlinearity from the thin layer of the waveguide [14, 15]. In Fig. 1a, this layer is marked by the dot lines. The elimination should be compensated by additional tensions $\delta \sigma_{13}, \delta \sigma_{33}$, and, respectively, the 
additional parts of the velocity of the medium $\delta v_{x}, \delta v_{z}$ within this layer. Because the layer is thin, the dominating derivative is $\partial / \partial z$. It is possible to write down

$$
\delta \sigma_{13} \approx-\sigma_{13}^{\mathrm{NL}}
$$

where

$$
\delta \sigma_{13}=\rho s_{\mathrm{t}}^{2}\left[\frac{\partial\left(\delta u_{x}\right)}{\partial z}+\frac{\partial\left(\delta u_{z}\right)}{\partial x}\right] \approx \rho s_{\mathrm{t}}^{2} \frac{\partial\left(\delta u_{x}\right)}{\partial z} .
$$

This makes possible to calculate $\delta v_{x}$ :

$$
\begin{aligned}
& \frac{\partial\left(\delta u_{x}\right)}{\partial z} \approx-\frac{1}{v_{0}} \frac{\partial\left(\delta u_{x}\right)}{\partial \eta} \approx-\frac{\sigma_{13}^{\mathrm{NL}}}{\rho s_{\mathrm{t}}^{2}}, \\
& \delta v_{x} \equiv \frac{\partial\left(\delta u_{x}\right)}{\partial t} \approx \frac{\partial\left(\delta u_{x}\right)}{\partial \eta} \approx v_{0} \frac{\sigma_{13}^{\mathrm{NL}}}{\rho s_{\mathrm{t}}^{2}} .
\end{aligned}
$$

As the result, the expression for the additional parts of the velocity is $\delta v_{x}=v_{0} \sigma_{13}^{\mathrm{NL}} / \rho s_{\mathrm{t}}^{2}$, and analogously $\delta v_{z}=v_{0} \sigma_{33}^{\mathrm{NL}} / \rho s_{1}^{2}$. When the nonlinearity is moderate, the additional term is small for the dominating component of the velocity $v_{x}$ but gives an essential correction to the small component $v_{z}$.

To derive the equations for the slowly varying amplitudes $A_{j}(z, y)$, the reciprocity relation has been used $[13,16]:$

$$
\begin{aligned}
& \frac{\partial}{\partial t}\left[\rho \frac{\partial u_{i}^{(1)}}{\partial t} \frac{\partial u_{i}^{(2)^{*}}}{\partial t}+c_{i j k l} u_{i j}^{(1)} u_{k l}^{(2)^{*}}\right] \\
& \quad=\frac{\partial}{\partial x_{j}}\left[\left(c_{i j k l} u_{k l}^{(1)}+\sigma_{i j}^{\mathrm{NL}}\right) \frac{\partial u_{i}^{(2)^{*}}}{\partial t}+c_{i j k l} u_{k l}^{(2)^{*}} \frac{\partial u_{i}^{(1)}}{\partial t}\right] \\
& \quad-\sigma_{i j}^{n l} \frac{\partial}{\partial t} \frac{\partial u_{i}^{(2)^{*}}}{\partial x_{j}}, \\
& \frac{\partial \boldsymbol{u}_{j}^{(1)}}{\partial t}=\frac{c_{\mathrm{t}}}{2} A_{j}(z, y) \boldsymbol{f}_{j}(x) \mathrm{e}^{\mathrm{i} \omega_{j} \eta}, \\
& \frac{\partial \boldsymbol{u}_{j}^{(2)^{*}}}{\partial t}=c_{\mathrm{t}} \boldsymbol{f}_{j}^{*}(x) \mathrm{e}^{-\mathrm{i} \omega_{j} \eta},
\end{aligned}
$$

where $\boldsymbol{u}_{j}^{(1)}$ are the positive frequency component of the mechanical displacement of the nonlinear wave of the frequency $\omega_{j} ; \boldsymbol{u}_{j}^{(2)^{*}}$ are the negative frequency component of the monochromatic linear wave; $c_{i j k l}=\rho_{1} c_{\mathrm{t}}^{2}\left(\delta_{i k} \delta_{j l}+\right.$ $\left.\delta_{i l} \delta_{j k}\right)+\rho_{1}\left(c_{1}^{2}-2 c_{\mathrm{t}}^{2}\right) \delta_{i j} \delta_{k l}$ for the isotropic film (analogously for the substrate), $\sigma_{i j}^{n l}$ are the components of the nonlinear part of the Piola-Kirchhoff tensor $[1,3,15]$. The normalization of the transverse profiles $\boldsymbol{f}_{j}$ has been chosen to the unity value of the flux of the energy of the mode. The integration of Eq. (5) over the transverse coordinate $x$ results in the orthogonality relations for the linear eigenmodes and makes possible to derive the coupled equations for the slowly varying amplitudes in the nonlinear case.

Let us note that the nonlinearity in the boundary conditions matches with the volume nonlinearity and cannot be considered separately. Earlier there was an attempt to reduce Eq. (1) to the set of two weakly coupled equations for slowly varying amplitudes that depend on the running time $\eta$ and the longitudinal coordinate $z$ [17].
But it seems incorrect for the surface Rayleigh-like waves, because the different components of the mechanical displacement of the surface wave are strongly coupled due to the boundary conditions even in the linear case. Moreover, in [17] the used equations did not have any correct correspondence to the linear case. The experimental results [18] were devoted to the nonlinear periodic waves with the essential dispersion, whose dynamics differs from the pulse dynamics. Also the nonlinear modules for the film and the substrate used in [18] were essentially different, in a contrast to the case considered here.

The wave dynamics does not depend on the choice of the parameter $T$ for the enough large values of $T$ and for a number of used harmonics more than 200. Namely, several simulations with the same parameters have been realized with different values of $T$, and the results have been independent of the value of $T$. An alternative equivalent approach to derive the equations for amplitudes of harmonics is based on the variational principle with averaged Hamiltonian or Lagrangian function of the elastic field $[2,3,10]$.

The propagation of the baseband nonlinear waves is described by a set of coupled ordinary differential equations for harmonics [6], and the total number of harmonics is of about 256-4096. The splitting with respect to physical factors has been applied. Namely, at the first half-step the nonlinearity, the wave dispersion, and the dissipation has been taken into account. At the second half-step the wave diffraction has been considered. In the simulations the parabolic approximation have been applied

$$
\begin{aligned}
& \frac{\partial A_{j}}{\partial z}=-\mathrm{i}\left(k_{j}-j k_{1}\right) A_{j}-\Gamma_{j} A_{j} \\
& \quad+\mathrm{i} \omega_{j}\left(\sum_{m<j} P_{m, j} A_{m} A_{j-m}\right. \\
& \left.\quad+2 \sum_{m>0} P_{m, m+j} A_{m+j} A_{m}^{*}\right)- \text { the first half-step, } \\
& \frac{\partial A_{j}}{\partial z}=-\frac{\mathrm{i}}{2 k_{j}} \frac{\partial^{2} A_{j}}{\partial y^{2}} \text { - the second half-step. }
\end{aligned}
$$

Here $k_{j} \equiv k\left(\omega_{j}\right)$ is determined from linear dispersion equation; $\Gamma_{j}=j^{2} \Gamma_{1}$ is the dissipation of the $j$-th harmonic. In simulations it has been taken $\Gamma(\omega=$ $\left.10^{9} \mathrm{~s}^{-1}\right)=0.2 \mathrm{~cm}^{-1}$. The coefficients $P_{m, j}$ are expressed through the linear and nonlinear modules of two contacting media and, due to their complexity, they are not presented here. These coefficients have been calculated numerically. Using the reciprocity relation, the nonlinear boundary conditions for the stresses at the interfaces are taken into account simultaneously, and there is no necessity to separate the nonlinearity in the volume equations and in the boundary conditions here, in a distinction to [11].

The linear dispersion of the considered waves corresponds to so-called Benjamin-Ono (B-O) equation. In the case of the model described by the solitonic $\mathrm{B}-\mathrm{O}$ equation all the coefficients $P_{m, j}$ are equal [19]. 

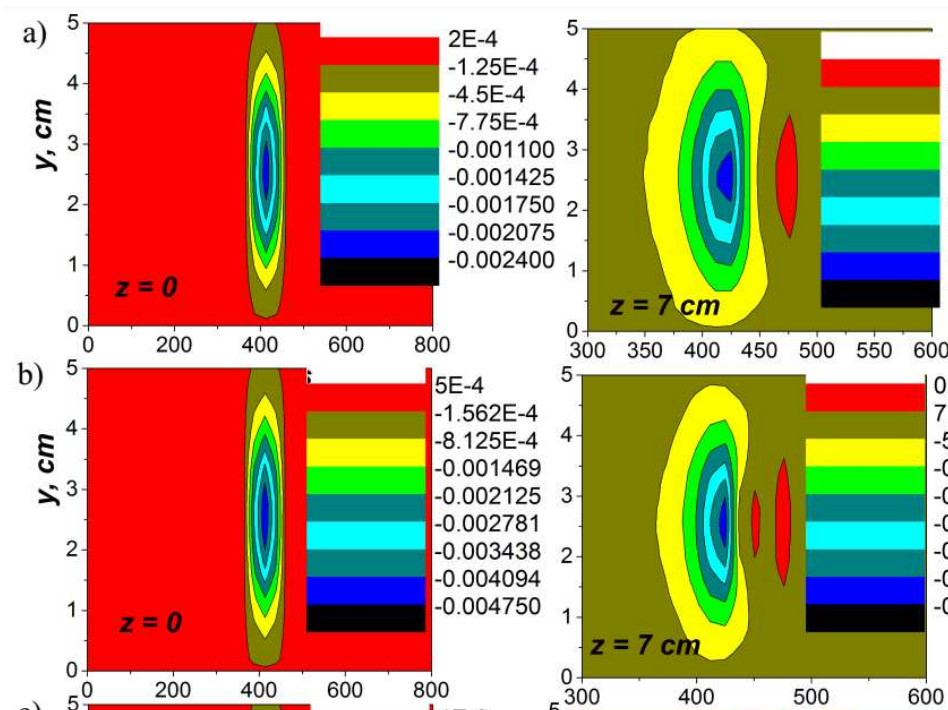

c
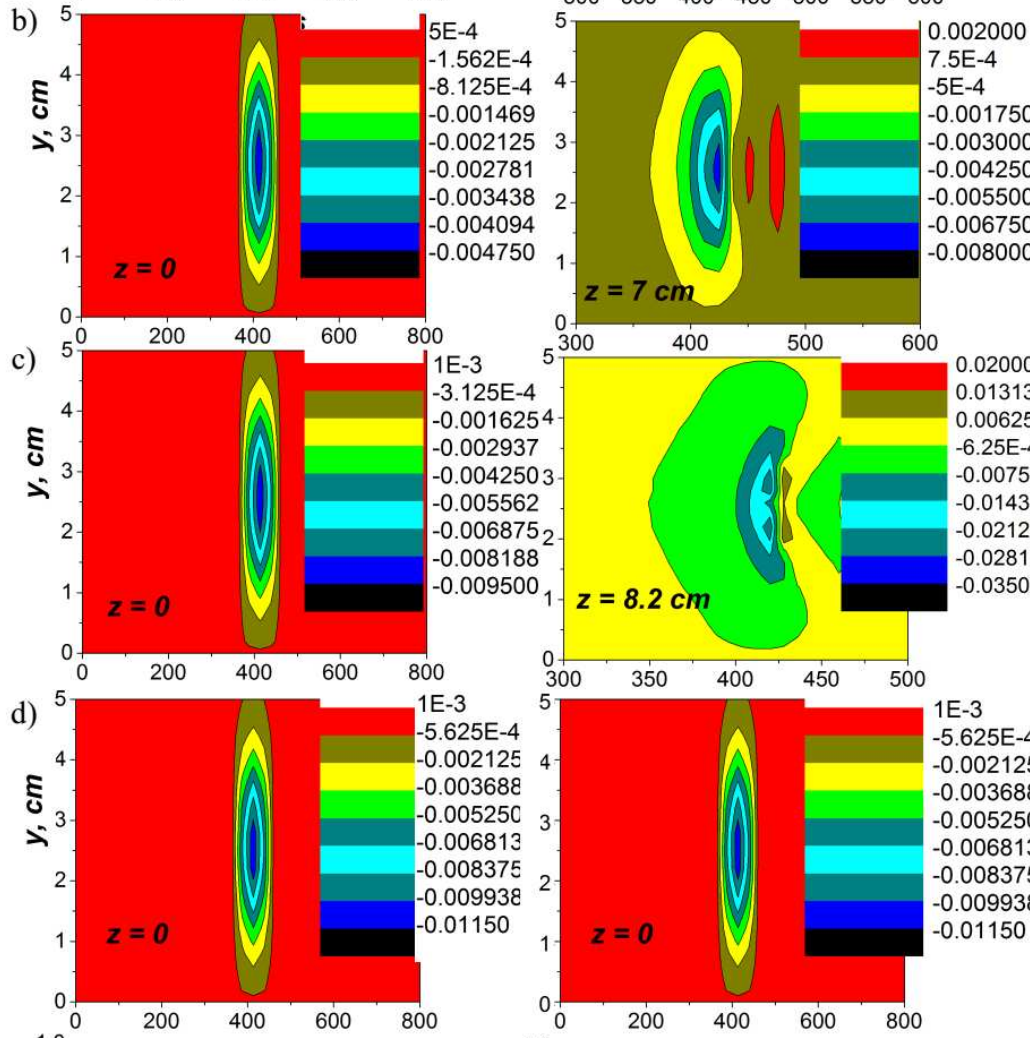

0.02000

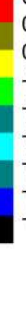

0.01313
0.006251
$-6.25 E-4$

$-6.25 \mathrm{E}-4$
-0.00750

$-0.01438$

$-0.02125$

-0.02813
-0.03500
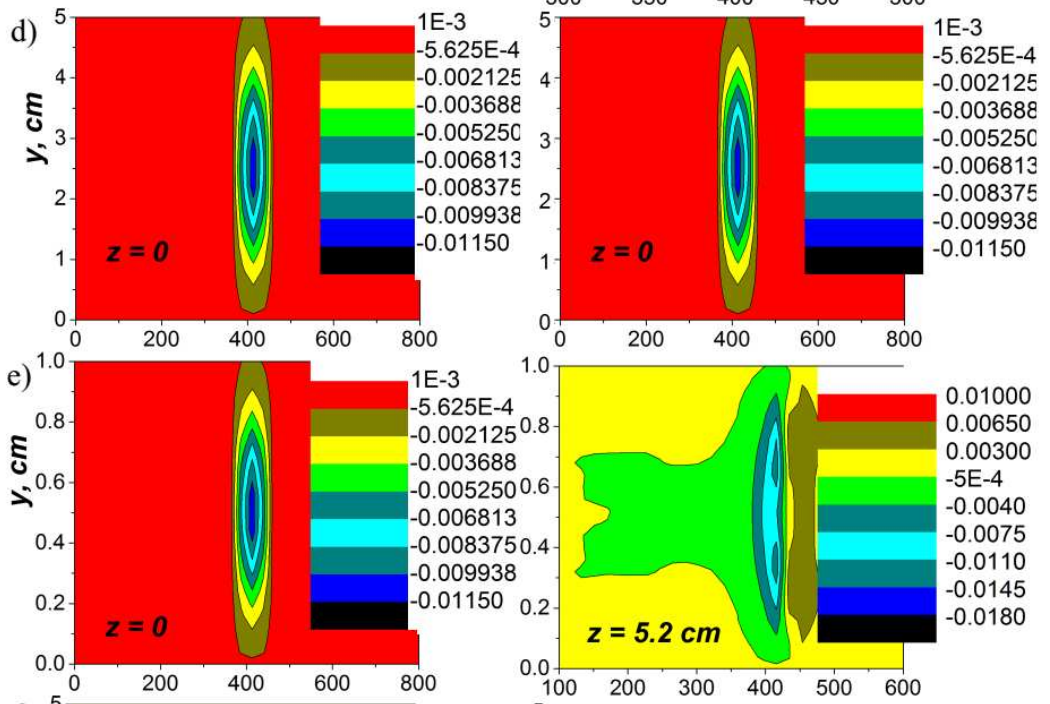

$1 \mathrm{E}-3$

$-5.625 \mathrm{E}-4$

$-0.002125$

$-0.003688$

$-0.00525 \mathrm{C}$

$-0.006813$

$-0.008375$

$-0.009938$

$-0.01150$
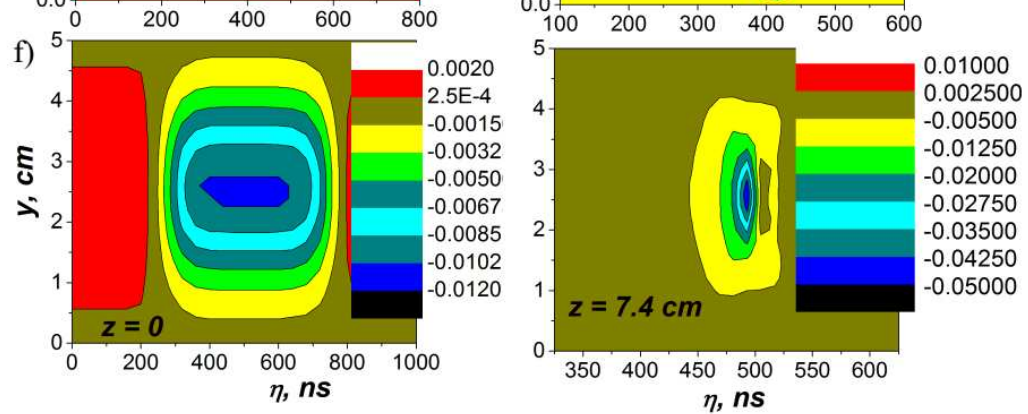
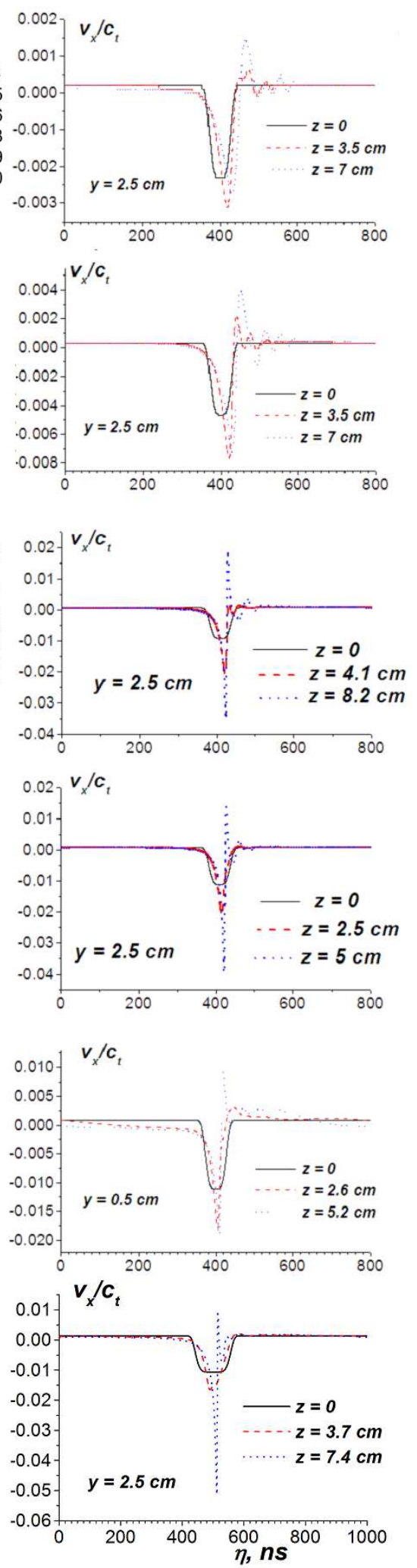

Fig. 2. The dynamics of baseband pulses in $\mathrm{Si}(\mathrm{Ge}) \mathrm{O}_{2}-\mathrm{Si}$ structure. Part (a) is for the extreme value of the input pulse $v_{x 0} / c_{\mathrm{t}}=-0.0025 ;$ (b) $v_{x 0} / c_{\mathrm{t}}=-0.005 ;$ (c) $v_{x 0} / c_{\mathrm{t}}=-0.01 ;$ (d) $v_{x 0} / c_{\mathrm{t}}=-0.012 ;$ (e) $v_{x 0} / c_{\mathrm{t}}=-0.012$, but the narrower initial pulse; (f) $v_{x 0} / c_{\mathrm{t}}=-0.012$, but the wider initial pulse. The thickness of the film is $h=0.25 \mu \mathrm{m}$. 
In our case they increase with frequency growth. Physically it is due to the concentration of the acoustic energy near the surface when the propagating pulse becomes shorter. The numerical simulations have shown that the baseband nonlinear waves in this system with above-pointed nonlinearity differ from the $\mathrm{B}-\mathrm{O}$ solitons. The used nonlinear modules are $A=B=-3 \times 10^{12} \mathrm{dyn} / \mathrm{cm}^{2}$, $C=-5 \times 10^{12} \mathrm{dyn} / \mathrm{cm}^{2}[1,2]$.
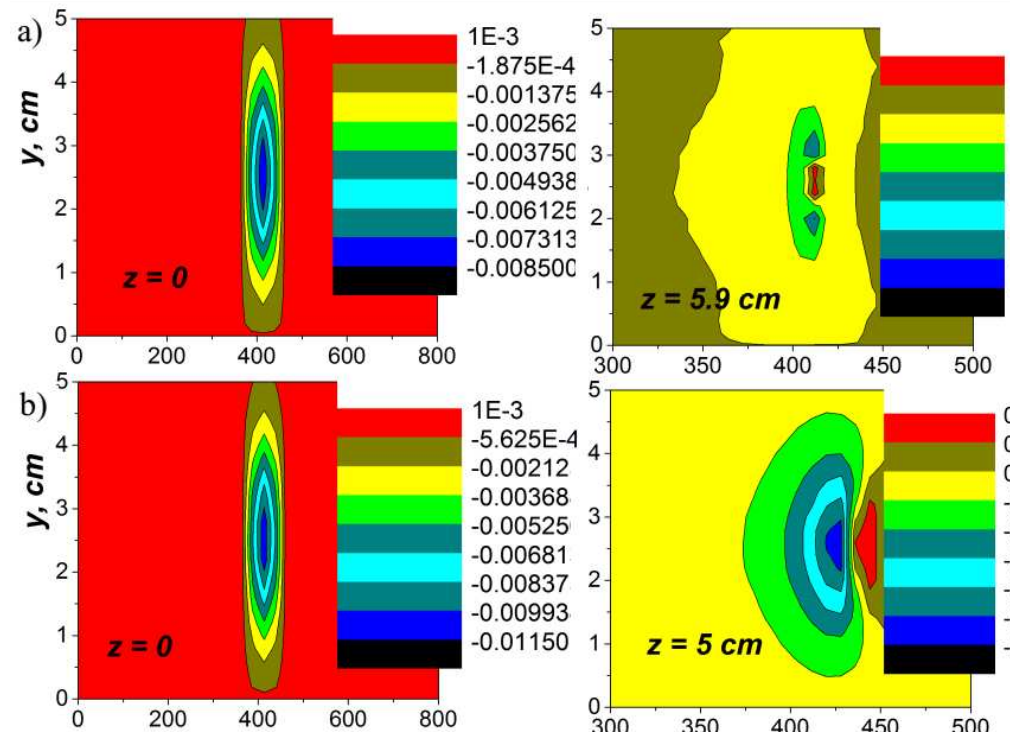

$1 \mathrm{E}-3$

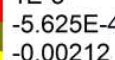

$-0.00212$ $-0.00368$ $-0.00525$ -0.00681
-0.008372 $-0.00993$ $-0.01150$
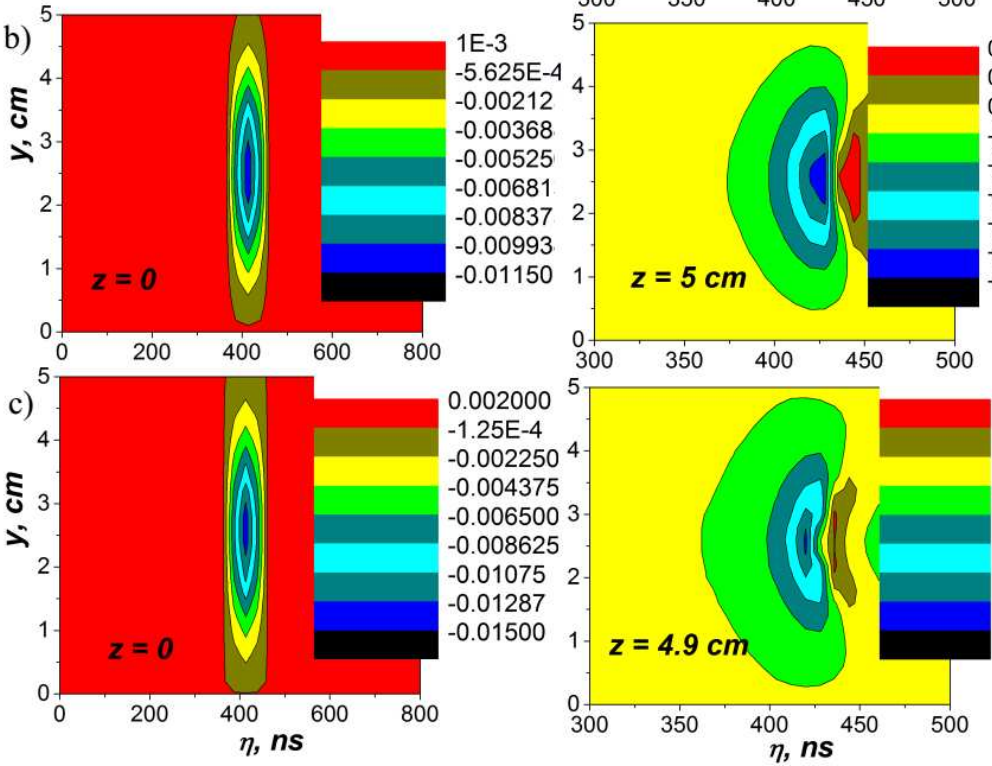

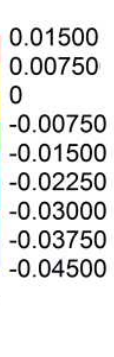

0.01000 0.005938 0.001875
-0.002187 $-0.006250$ $-0.01031$ $-0.01437$ $-0.01844$ $-0.02250$

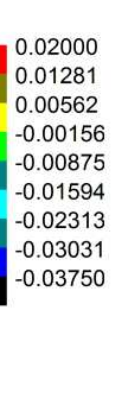

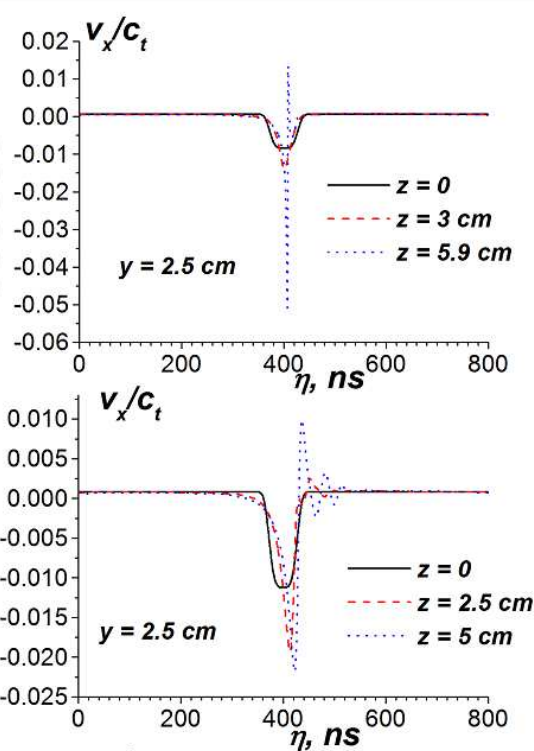

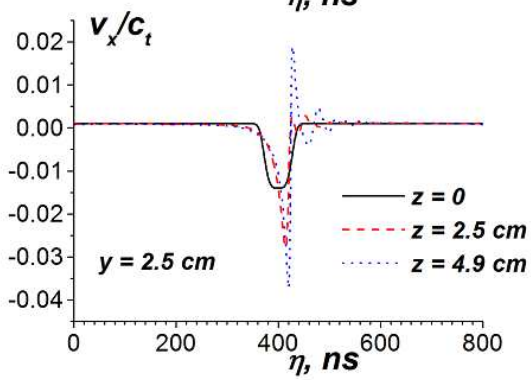

Fig. 3. The dynamics of baseband pulses in $\mathrm{Si}(\mathrm{Ge}) \mathrm{O}_{2}-\mathrm{Si}$ structure. In part (a) the thickness of the film is $h=0.1 \mu \mathrm{m}$, $v_{x 0} / c_{\mathrm{t}}=-0.009$; (b) the thickness of the film is $h=0.5 \mu \mathrm{m}, v_{x 0} / c_{\mathrm{t}}=-0.012$; (c) the thickness of the film is $h=0.5 \mu \mathrm{m}$, $v_{x 0} / c_{\mathrm{t}}=-0.015$.

In the simulations the generation of the nonlinear baseband waves from an initial almost rectangular pulse at $z=0$ has been investigated, see Figs. 2, 3. The dependences of the vertical component of the velocity of the free surface of the film $v_{x}(x=0)$ are presented on the running time $\eta$. The first parts are the initial distributions $z=0$ of the vertical component of velocity at the free surface of the film $v_{x}(x=0, \eta, y) / c_{\mathrm{t}}$ in the plane $(\eta, y)$, the second parts are the distributions of $v_{x} / c_{\mathrm{t}}$ at the maximum compression of the pulse; the third parts are the initial (solid line), intermediate (dash line), and final (dot line) distributions of $v_{x} / c_{\mathrm{t}}$ in the center $y=L_{y} / 2$. The final distributions in the third part correspond to the maximum compression of the pulse, as in the second part. The velocity of the elastic medium is normalized to the transverse sound bulk velocity of the film $c_{\mathrm{t}}=2.5 \times 10^{5} \mathrm{~cm} / \mathrm{s}$. In all figures $v_{x 0}$ is the extreme value of the input pulse at $z=0$.
Let us note that there exists a self-similar solution of Eqs. (6), which describes a traveling nonlinear wave with the constant velocity of propagation $[6,10]$. But the set of equations for the nonlinear SAW is not fully integrable, and it is impossible to conclude that such a solitary traveling wave must be excited from each initial data of enough large value [19]. It is impossible also to separate the solitonic and non-solitonic parts of the nonlinear wave there, because of absence of true solitons.

The nonlinear baseband wave possesses the certain asymmetry under its propagation. The polarity of the nonlinear pulse depends on the signs of the quadratic nonlinear modules. A stable true solitary wave is not formed during the evolution of input long rectangular pulses, as our simulations have been demonstrated. The nonlinear baseband wave manifests rather like a shock-like pulse. From a long initial rectangular pulse or a sequence of two neighboring pulses only a single shock- 
-like pulse is formed. When the input rectangular pulse is taken enough long (Fig. 2c,d,f), it is possible to get the nonlinear pulses of large peak values, where an approximation of moderate nonlinearity ceases to be valid. To generate the nonlinear pulse with the duration $\leq 3 \mathrm{~ns}$ it is necessary to use the structures with the thickness of the film $h<0.5 \mu \mathrm{m}$.

The results of the pulse dynamics with thicknesses of the film $h=0.1 \mu \mathrm{m}$ and $h=0.5 \mu \mathrm{m}$ are given in Fig. 3 . When the thickness of the film is smaller $h \approx 0.1 \mu \mathrm{m}$, the wave dispersion is lower and the single shock is formed at smaller distances. In the structures with thicker films $h \geq 0.5 \mu \mathrm{m}$ the wave dispersion can dominate and prevents the formation of shocks. This difference can be explained in the following manner. In the structures with the thinner films $h \leq 0.25 \mu \mathrm{m}$ the surface wave penetrates deeply into the substrate. In the structures with thicker films $h \geq 0.5 \mu \mathrm{m}$ the surface wave is localized near the interface of the film-substrate.

The frequency range of the pulse under maximum compression (at the distances $z \approx 5-10 \mathrm{~cm}$ ) lies within the interval $0<\omega \leq 5 \times 10^{9} \mathrm{~s}^{-1}$. Therefore, the influence of the wave diffraction is important, when the transverse half-width of the initial pulse is $\leq 0.2 \mathrm{~cm}$, see Fig. 2e.

The similar dynamics of the nonlinear wave excitation has been obtained for different values of nonlinear parameters $A, B, C$, because the values of nonlinear coefficients $P_{m, j}$ are not constant for any set of nonlinear parameters of the system film-substrate. Let us note that in the case of the fully integrable Benjamin-Ono or the Korteveg-de Vries solitonic systems it is possible to excite several solitons from a long initial pulse [17].

\section{The focused monopulses}

It is possible to focus the pulses, when the arc-like antenna is used for the excitation of initial rectangular acoustic waves, see Fig. 1d. Earlier the focusing of nonlinear waves was investigated for the envelope nonlinear pulses [20].
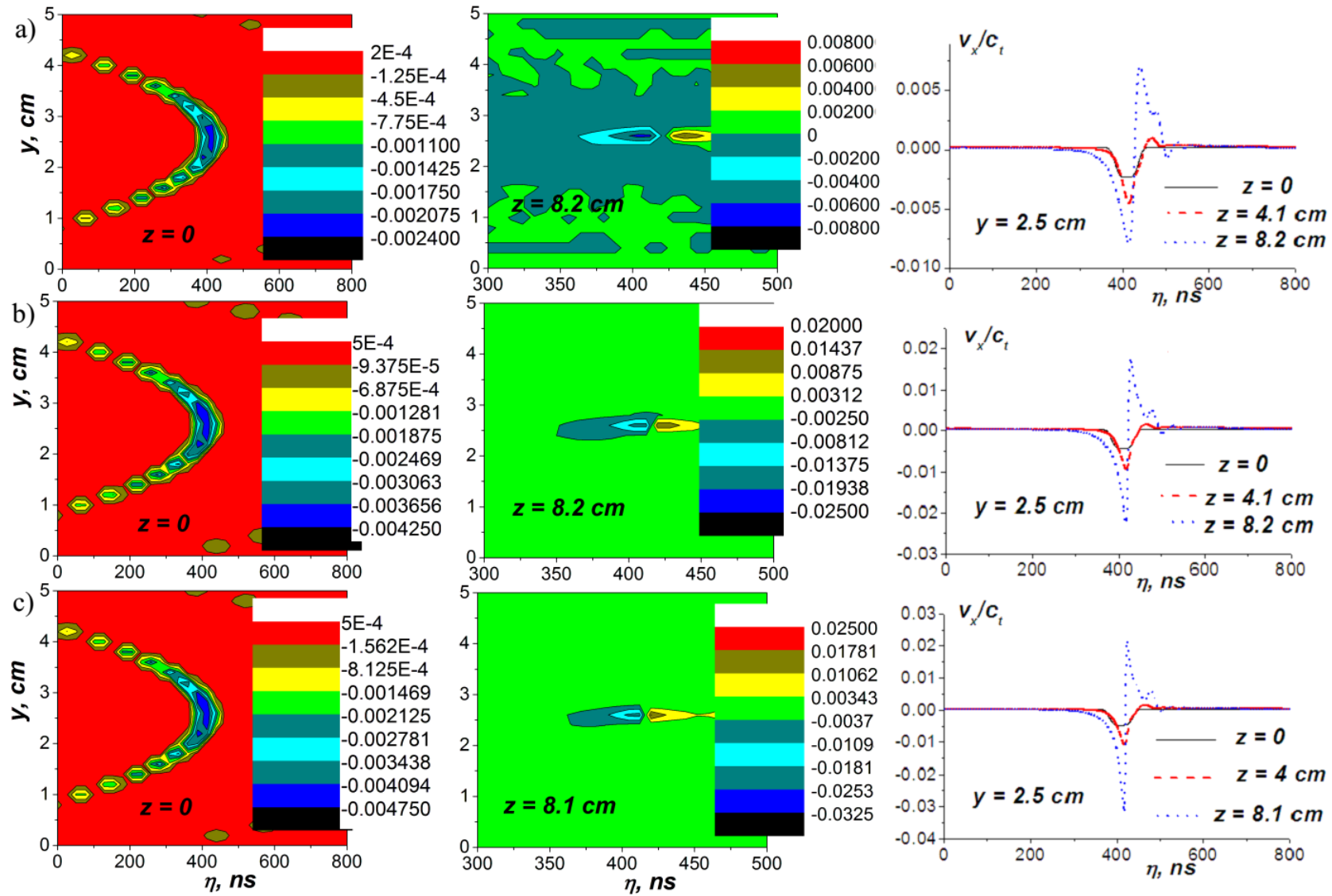

Fig. 4. The dynamics of the focused baseband pulses in $\mathrm{Si}(\mathrm{Ge}) \mathrm{O}_{2}-\mathrm{Si}$ structure. Part (a) is for $v_{x 0} / c_{\mathrm{t}}=-0.0025$; (b) is for $v_{x 0} / c_{\mathrm{t}}=-0.0045,(\mathrm{c})$ is for $v_{x 0} / c_{\mathrm{t}}=-0.005$. The thickness of the film is $h=0.25 \mu \mathrm{m}$.

It is used the same method for the baseband waves. In the plane $z=0$ the corresponding phase shift has been calculated for all harmonics $A_{j}, j=1,2, \ldots$ The curvilinear shape of the initial pulse in the plane $(\eta, y)$ at 
$z=0$ is due to the delay of propagation of the wave from the central part of the antenna, see Fig. 1d. For all the cases the focusing distance is $R=7.5 \mathrm{~cm}$. The results of simulations are given in Figs. 4, 5. The positions of three parts are the same as for Figs. 2, 3. In Fig. 4 it is seen that the focusing of monopulses leads to essential increase of the nonlinear shock waves. In Fig. 4c and Fig. 5, at near the focus point the nonlinearity is no longer moderate.
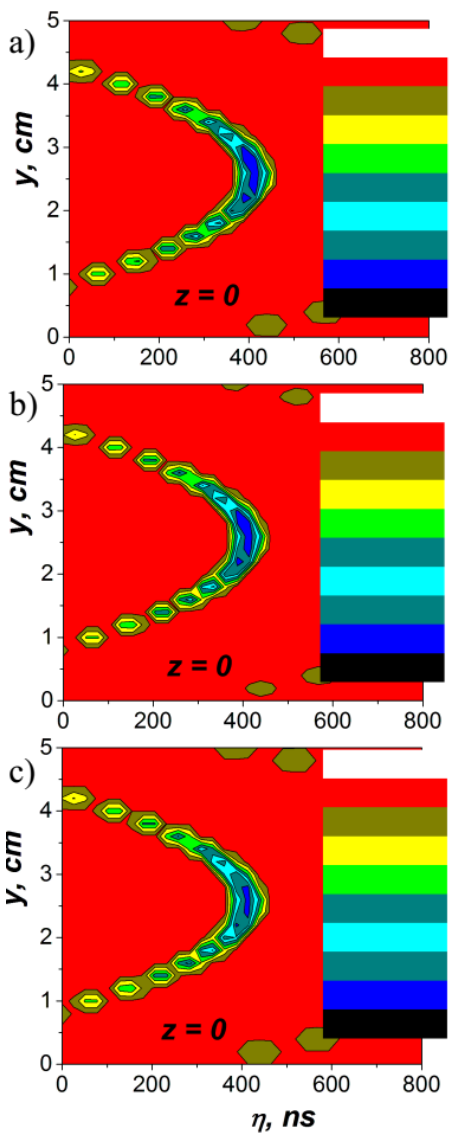
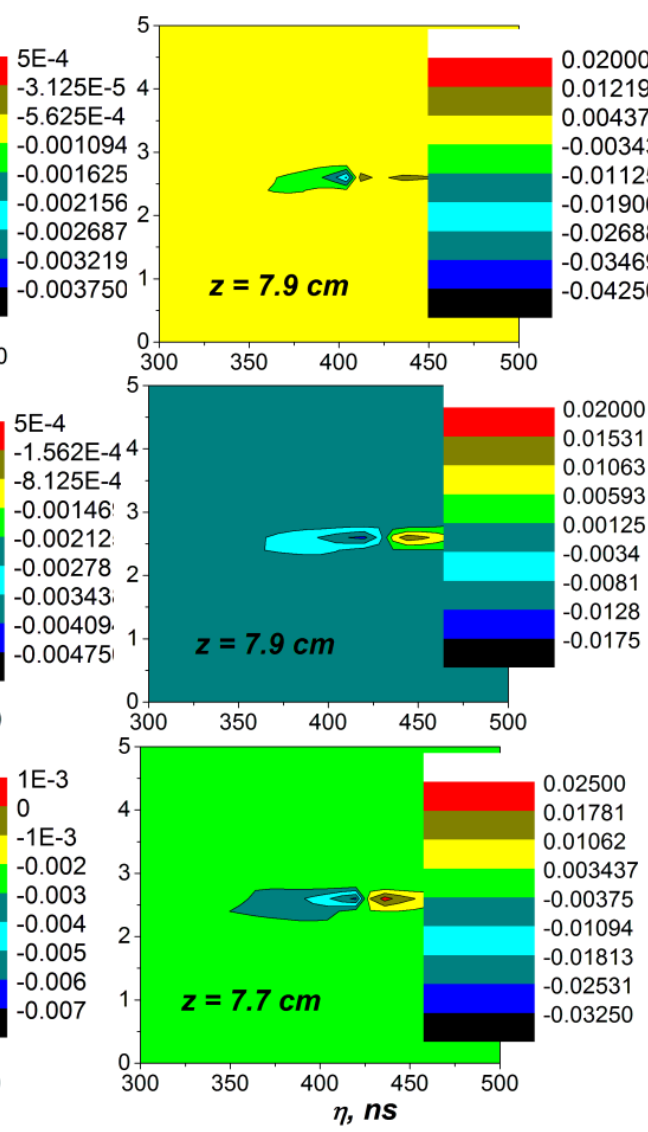

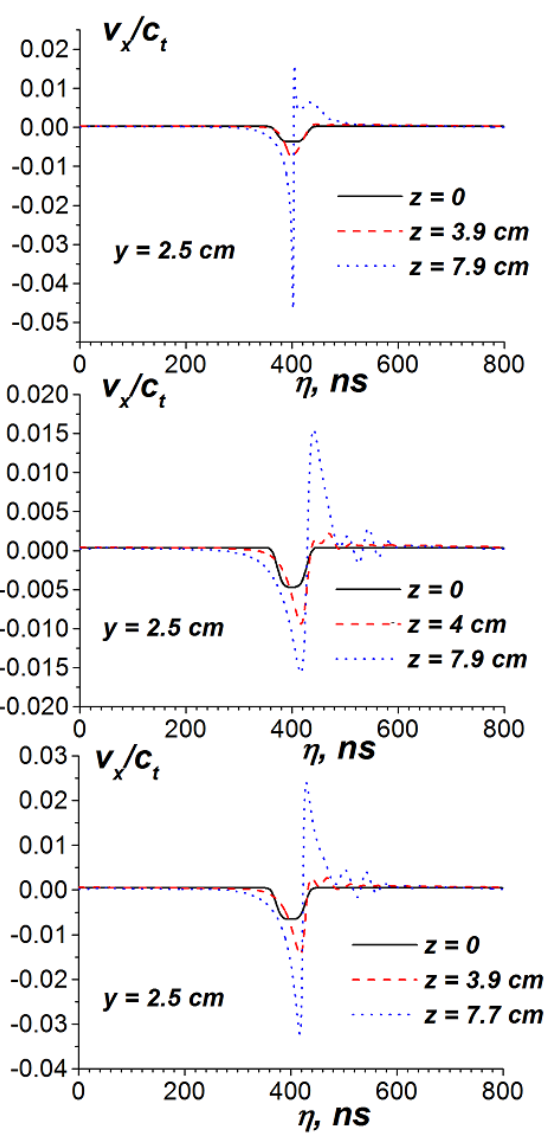

Fig. 5. The dynamics of the focused baseband pulses in $\mathrm{Si}(\mathrm{Ge}) \mathrm{O}_{2}-\mathrm{Si}$ structure. Part (a) is for the thickness of the film $h=0.1 \mu \mathrm{m}, v_{x 0} / c_{\mathrm{t}}=-0.004 ;$ (b) is for the thickness of the film $h=0.5 \mu \mathrm{m}, v_{x 0} / c_{\mathrm{t}}=-0.005$, (c) is for the thickness of the film $h=0.5 \mu \mathrm{m}, v_{x 0} / c_{\mathrm{t}}=-0.007$.

One can see that the nonlinear surface waves can be focused easier in the structures with the thinner films $h<0.25 \mu \mathrm{m}$, compare Fig. 4c and Fig. 5a. In that turn, the structures with the thicker films $h \geq 0.5 \mu \mathrm{m}$ possess an essential wave dispersion, which can prevent the creation of the nonlinear focus, as our simulations have shown.

\section{Conclusions}

The excitation of nonlinear acoustic monopulses, or baseband waves, in the gigahertz range is theoretically analyzed. The monopulses have a wide spectrum $\leq 1 \mathrm{GHz}$. Simulations of the baseband nonlinear wave dynamics in the layered system, which includes a film of a thickness $h=0.1-1 \mu \mathrm{m}$ and a half-infinite substrate, demonstrate a formation of shock-like pulses of surface acoustic waves. The dynamics of the baseband pulses is non-solitonic in these structures. It seems that a non-soliton behavior is a general feature of the monopulse waves in half-bounded systems. The transverse inhomogeneity in the plane of the film and the wave diffraction affect the dynamics of monopulses. It is possible to focus the monopulses and to produce strong shock waves where the nonlinearity ceases to be moderate.

The dynamics of the pulses of surface acoustic waves depends on the thickness of the film. Namely, when the thickness of the film is $h \leq 0.25 \mu \mathrm{m}$, the nonlinearity dominates over the wave dispersion. In the case of the thicker films $h \geq 0.5 \mu \mathrm{m}$ the wave dispersion can dominate. Therefore, the structures with the thicker films can be useful for observation of both true baseband solitons and the envelope ones.

The formation of short $\approx 1$ ns acoustic shock-like pulses is important for the signal processing and the acoustic treatment of interfaces in microelectronics. 


\section{References}

[1] K. Naugolnykh, L. Ostrovsky, Nonlinear Wave Processes in Acoustics, Cambridge University Press, Cambridge, UK 1998.

[2] A.P. Mayer, Phys. Rep. 256, 237 (1995).

[3] M.F. Hamilton, Yu.A. Il'inskii, E.A. Zabolotskaya, J. Acoust. Soc. Am. 105, 639 (1999).

[4] A.V. Porubov, A.M. Samsonov, Int. J. Non-Linear Mechanics 30, 861 (1995).

[5] Y.B. Fu, S.L.B. Hill, Wave Motion 34, 109 (2001).

[6] C. Eckl, A.S. Kovalev, A.P. Mayer, Phys. Rev. Lett. 81, 983 (1998).

[7] A.M. Lomonosov, P. Hess, Phys. Rev. Lett. 89, 095501 (2002).

[8] A.S. Kovalev, A.P. Mayer, C. Eckl, G.A. Maugin, Phys. Rev. E 66, 036615 (2002).

[9] A.M. Lomonosov, P. Hess, A.P. Mayer, Phys. Rev. Lett. 88, 076104 (2002).

[10] C. Eckl, A.S. Kovalev, A.P. Mayer, A.M. Lomonosov, P. Hess, Phys. Rev. E 70, 046604 (2004).
[11] Mingxi Deng, J. Phys. D, Appl. Phys. 37, 1385 (2004).

[12] G.A. Maugin, Nonlinear Waves in Elastic Crystals, Oxford Univ. Press, New York 1999.

[13] B.A. Auld, Acoustic Fields and Waves in Solids, Vol. 2, Wiley, New York 1973.

[14] L.A. Vainshtein, Electromagnetic Waves, Radio i Svyaz', Moscow 1988 (in Russian).

[15] A.A. Barybin, Electrodynamics of Waveguiding Structures, Fizmatlit, Moscow 2007 (in Russian).

[16] D. Royer, E. Dieulesaint, Elastic Waves in Solids 2: Generation, Acousto-Optic Interaction, Applications, Springer-Verlag, New York 1999.

[17] V.I. Nayanov, Multifield Solitons, Fizmatlit, Moscow 2006 (in Russian).

[18] V.I. Nayanov, JETP Lett. 44, 314 (1986).

[19] M. Ablowitz, H. Segur, Solitons and the Inverse Scattering Transform, SIAM, Philadelphia 1981.

[20] V. Grimalsky, S. Koshevaya, O. Kolokoltsev, Acta Phys. Pol. A 119, 528 (2011). 